\title{
The origin of giant ferroelectric distortion and the nature of pressure-induced spin-state transition in $\mathrm{BiCoO}_{3}$
}

Multiferroic materials, which have coexisting magnetism and ferroelectricity, are of great technological and fundamental importance. Researchers from the Key Laboratory of Materials Physics, Institute of Solid State Physics, Chinese Academy of Sciences, Hefei, China have sought the origin of the giant tetragonal ferroelectric distortion and the nature of the pressure-induced spin-state transition in the potentially multiferroic material $\mathrm{BiCoO}_{3}$. The present study, in strong contrast to some recent works, provides an insight into this potentially multiferroic material.

$\mathrm{BiCoO}_{3}$ was recently synthesized by high-pressure (HP) technique; it has been suggested to be a promising multiferroic material by Uratani et al. and Ravindran et al. through first-principles Berry-phase calculations. $\mathrm{BiCoO}_{3}$ has a giant tetragonal lattice distortion of $c / a=$ 1.27 with remarkable off-center atomic displacements. It was proposed that the giant tetragonal distortion originates from the lifting of the orbital degeneracy of the high spin (HS) $(S=2) \mathrm{Co}^{3+}$ ions and is stabilized by the subsequent $x y$-type ferro-orbital ordering. Note that orbitally degenerate transition-metal oxides quite often display an orbital ordering (OO) but ferroelectric (FE) materials from them are rare as ferroelectricity and magnetism seem to, and actually do, in most cases, exclude each other. Therefore, the proposed mechanism for the giant FE distortion does not appear to be straightforward. Note also that controversial spin states, both low-spin (LS) and intermediate-spin (IS) $(S=1)$, were suggested for the HP phase.

This work describes the attempts of Dr. Jia and Prof. Zeng et al., in cooperation with Prof. Wu from Germany and Prof. Lin from Hong Kong, China, to find the origin of the giant tetragonal $\mathrm{FE}$ distortion in the ambient phase of $\mathrm{BiCoO}_{3}$. They have identified the nature of the pressure-induced spin-state transition, using two sets of configuration-state-constrained generalized gradient approximation plus Hubbard $\mathrm{U}$ $(\mathrm{GGA}+\mathrm{U})$ calculations. Their results show that the giant tetragonal distortion is driven by a strong Bi-O covalency (rather than by the aforementioned lifting of orbital degeneracy) and is further stabilized by an $x y$-type $\mathrm{OO}$ of the $\mathrm{HS} \mathrm{Co}^{3+}$ ions. Moreover, they find that the pressure-induced spin-state transition is via a mixed HS-LS state, and that the transition would be complete upon a large volume decrease of about $20 \%$ (under about $8 \mathrm{GPa}$ ). The mixed HS-LS state accounts well for the available experimental results.

See the article: Jia T, Wu H, Zhang G R, et al. Ab initio study of the giant ferroelectric distortion and pressure-induced spin-state transition in $\mathrm{BiCoO}_{3}$. Phys Rev B, 2011, 83: 174433

Open Access This article is distributed under the terms of the Creative Commons Attribution License which permits any use, distribution, and reproduction in any medium, provided the original author(s) and source are credited. 